\title{
Perfectly imperfect
}

\author{
Defects in nanostructures have their advantages.
}

There are many examples in nanotechnology of materials with near-perfect order, such as graphene, carbon nanotubes and singlecrystal nanowires. This perfection, which is the result of extensive research, as well as the natural tendency of nanoscale systems to be resistant to defects, has been exploited in devices such as sensors and transistors. However, many researchers are now trying to put defects back into these perfect structures - not to ruin them, but to make them even better.

This should not come as a surprise. Although the history of many new materials begins with a push towards purity, the effort to introduce impurities can start soon afterwards. The use of silicon in electronics, for example, was originally hampered because only metallurgical-grade silicon was available at the time, and it took several years of hard work before electronic-grade purity was reached (with a significant contribution from DuPont, which wanted to put it into white paint $\left.{ }^{1}\right)$. The ability to make a very pure form of silicon contributed to the triumph over its semiconducting rival, germanium.

But no sooner had impurities such as carbon and oxygen been removed from silicon than the race began to introduce other impurities: phosphorous to donate electrons, boron to donate holes, and so on. And as transistors shrank, the numbers of these implanted impurities dropped to hundreds per transistor or less, making the position of individual dopants matter ${ }^{2,3}$, and raising the prospect of 'engineering' individual defects into devices.

Examples of nanoscale defect engineering in graphene and diamond are reported in this issue of Nature Nanotechnology. Graphene has unique properties because of the perfection of its structure - electrons can travel through its hexagonal carbon lattice so easily that they effectively have no mass. Such perfection is an attractive feature because it leads to very high conductivities and novel quantum effects. But careful disruptions to that perfection can also be attractive, for example by allowing the steering or trapping of electrons. This kind of reasoning has prompted theorists to devote considerable attention to introducing defects in graphene.

On page 326 of this issue, Matthias Batzill and colleagues report the first experimental demonstration of a line defect in graphene. The defect forms spontaneously at the boundary between graphene domains that are defined by how they arrange themselves on a nickel substrate, and has a density of states that is reminiscent of the metallic edge states in graphene flakes. This suggests that it might function as a metallic wire. Additional functions could be derived from more complex defects (see page 316). There have also been reports that defects can lead to magnetism in pure carbon ${ }^{4}$, which suggests that they may be useful for spintronic devices.

On page 345, James Rabeau and colleagues focus on nitrogen-vacancy (NV) colour centres. These are defects that consist of a nitrogen atom added to the diamond lattice, next to a vacancy created by the removal of a carbon atom. The photoluminescent properties of NV centres have been studied since the 1970s, but room-temperature light emission from a single NV centre in bulk diamond was not observed until 1997 (ref. 5). Rabeau and colleagues observed intermittent light emission (also known as blinking) from individual $\mathrm{NV}$ centres in discrete 5-nm nanodiamonds, showing that such defects can be trapped in extremely small nanoparticles (see page 314). The advantage of isolating defects in such nanoparticles is that they could be used to probe their local environment. However, as blinking is a disadvantage for some applications, Rabeau and co-workers also developed a method to switch it off.

Defects are also being engineered into noncarbon nanostructures. Physicists working on quantum computing, for example, have launched a search for defects with properties similar to NV centres in diamond ${ }^{6}$. Other researchers have shown that the defects and stacking faults in the lattice of an InAs nanowire, which are usually distributed randomly, can be controlled to create a superlattice. The same approach can also be used to define quantum dots in nanowires ${ }^{8}$.

Remarkably, all of these defect-engineered systems rely only on a rearrangement of the atoms that are already present in the structure, rather than the introduction of dopants of a different element. Aside from reducing any toxicity associated with dopant atoms, this simplifies the fabrication process because it is no longer necessary to worry about implantation energies and damage, lattice matching or dopant positioning. Defects, it seems, are here to stay.

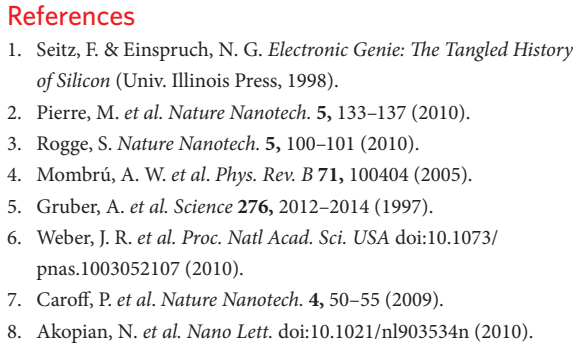

\section{The laser at fifty}

\section{Devices of all shapes and sizes are capable of lasing.}

What can you say about the laser that has not been said before? Not that it was a solution looking for a problem, nor that it has a central role in everyday life through CDs, DVDs and fibre-optic communications - although both of these statements are true. Ever since Ted Maiman got the first laser to work on 16 May 1960, by coaxing deep-red light from a ruby crystal coated on two parallel faces with silver and irradiated by a high-power flash lamp, lasers have become both bigger - the 192 beams of the National Ignition Facility occupy a building that is roughly the size of three football pitches - and smaller, in the form of various single-atom lasers. There has also been an explosion of interest in nanoscale lasers: it might be thought that the diffraction limit would hamper efforts to make solidstate lasers from nanowires, nanoparticles and the like, but researchers have overcome this problem by exploiting the interactions between photons and plasmons in various nanolasers. So let us celebrate 50 years of the laser, and look forward to many more. 